\title{
Phase Compensation Enhancement of Photon Pair Entanglement Generated from Biexciton Decays in Quantum Dots
}

\author{
Zong-Quan Zhou ${ }^{1,2}$, Chuan-Feng Li ${ }^{1, *}$, Geng Chen ${ }^{1}$, Jian-Shun Tang ${ }^{1}$, Yang Zou ${ }^{1}$, Ming Gong ${ }^{1}$ and Guang-Can Guo ${ }^{1}$ \\ ${ }^{1}$ Key Laboratory of Quantum Information, University of Science and Technology of China, CAS, Hefei, 230026, People's Republic of China \\ ${ }^{2}$ School of Instrumentation and Opto-electronics Engineering, \\ Hefei University of Technology, Hefei, 230009, People's Republic of China
}

(Dated: November 6, 2018)

\begin{abstract}
Exciton fine-structure splittings within quantum dots introduce phase differences between the two biexciton decay paths that greatly reduce the entanglement of photon pairs generated via biexciton recombination. We analyze this problem in the frequency domain and propose a practicable method to compensate the phase difference by inserting a spatial light modulator, which substantially improves the entanglement of the photon pairs without any loss.
\end{abstract}

PACS numbers: 03.67.Bg 78.55.Cr 42.50.-p

Entangled photon pairs play a crucial role in much of quantum information processing [1-4]. The most widely used methods for generating entangled photon pairs involve nonlinear optical processes, such as spontaneous parametric down conversion (SPDC) [5, 6]. However, high multi-photon probabilities and low quantum efficiencies associated with SPDC pose serious limitations on their applications in quantum information processing.

As an alternative, biexciton decays in single quantum dots (QDs) have been proposed as good sources of "on-demand" entangled photon pairs [7]. QDs also have the advantages of a mature fabrication technology and ease of integration into larger structures to make monolithic devices. However, "which-path" information provided by the fine-structure splitting (FSS) of the intermediate exciton state destroys the entanglement of photon pairs [8]. To overcome this problem, the energy splitting is tuned to near zero either by rapid thermal annealing [9], or optionally applying in-plane electric fields [10], magnetic fields [11, 12], uniaxial stresses [13] or light fields [14]. Such "triggered" entangled photon pair sources can also be engineered by simply selecting appropriate QDs with small FSSs [15], by energy-resolved post-selection [16], and by using highly-symmetric, site-controlled quantum dots grown in inverted pyramids [17].

In considering the photon emission distribution in the time domain, the two-photon state created in a QD is [18]

$$
\Psi(t)=\left(\sqrt{\frac{1}{\tau} e^{\frac{-t}{\tau}}} H_{X X} H_{X}+\sqrt{\frac{1}{\tau} e^{\frac{-t}{\tau}}} e^{i S t / \hbar} V_{X X} V_{X}\right) / \sqrt{2},
$$

where $S$ denotes the FSS energy, $t$ is the time delay between the first (biexciton) and the second (exciton) photon emission events, $\frac{1}{\tau} e^{\frac{-t}{\tau}}$ is the exciton photon emission probability distribution, and $\tau$ is the exciton lifetime. Thus time integration will reduce the overall degree of entanglement, and even lead to classically correlated states [19].

In this Report, we analyze this problem in the frequency domain and propose an optical arrangement to compensate

\footnotetext{
*email: cfli@ustc.edu.cn
}

the phase difference. Given the maximally entangled state $\left(H_{X X} H_{X}+V_{X X} V_{X}\right) / \sqrt{2}$, the fidelity is greatly improved and in our method is accompanied without photon losses, thus surpassing previous schemes that apply timing gates [19] and employ energy-resolved post selection [16].

By Fourier Transformation, we can re-express the twophoton state in the frequency domain

$$
\Psi(\omega)=\left(f_{H}(\omega) e^{i \varphi_{H}} H_{X X} H_{X}+f_{V}(\omega) e^{i \varphi_{V}} V_{X X} V_{X}\right) / \sqrt{2},
$$

with $f_{H}(\omega)=\left\{2 \pi \tau\left[1 /(2 \tau)^{2}+1 / \omega^{2}\right]\right\}^{-1 / 2}, \quad \varphi_{H}=$ $\tan ^{-1}(-2 \omega \tau), f_{V}(\omega)=\left\{2 \pi \tau\left[1 /(2 \tau)^{2}+1 /(S / \hbar-\omega)^{2}\right]\right\}^{-1 / 2}, \varphi_{V}=$ $\tan ^{-1}[2 \tau(S / \hbar-\omega)]$.

The polarization density matrix is given by

$$
\rho=\frac{1}{2}\left(\begin{array}{cccc}
1 & 0 & 0 & \alpha \\
0 & 0 & 0 & 0 \\
0 & 0 & 0 & 0 \\
\alpha^{*} & 0 & 0 & 1
\end{array}\right)
$$

with $\alpha=\int f_{H}(\omega) f_{V}(\omega) e^{i \varphi} d \omega$ and $\varphi=\varphi_{V}-\varphi_{H}$ is the phase difference. The fidelity with Bell state is

$$
f=\frac{1}{2}\left[1+\int f_{H}(\omega) f_{V}(\omega) \cos \varphi d \omega\right] .
$$

FSS limits the degree of entanglement in two ways, as illustrated in Figure 1. First, the phase difference between $\mathrm{H}$ - and V-polarized photons reduces the fidelity after time integration. Second, the overlap between their photon frequency distributions decreases as the FSS energy increases. We concentrate first on the phase difference.

Phase compensation is generally difficult to realize in the time domain, because it requires an accurate phase delay $(\sim S t / \hbar)$ rapidly varying with time. As shown in Fig. 1(b), the phase distribution is clearly a non-monotonic function of frequency, so it is also impossible to realize this compensation by simply using a dispersive element. The proposed experimental setup is shown in Fig. 2. The light from the QDs should initially be collimated and focused. For widely-used self-assembled QDs, the separation between biexciton (XX) and exciton (X) emission lines is generally several $m e V$ because of the biexciton binding energy [20], that then enables 
(a)

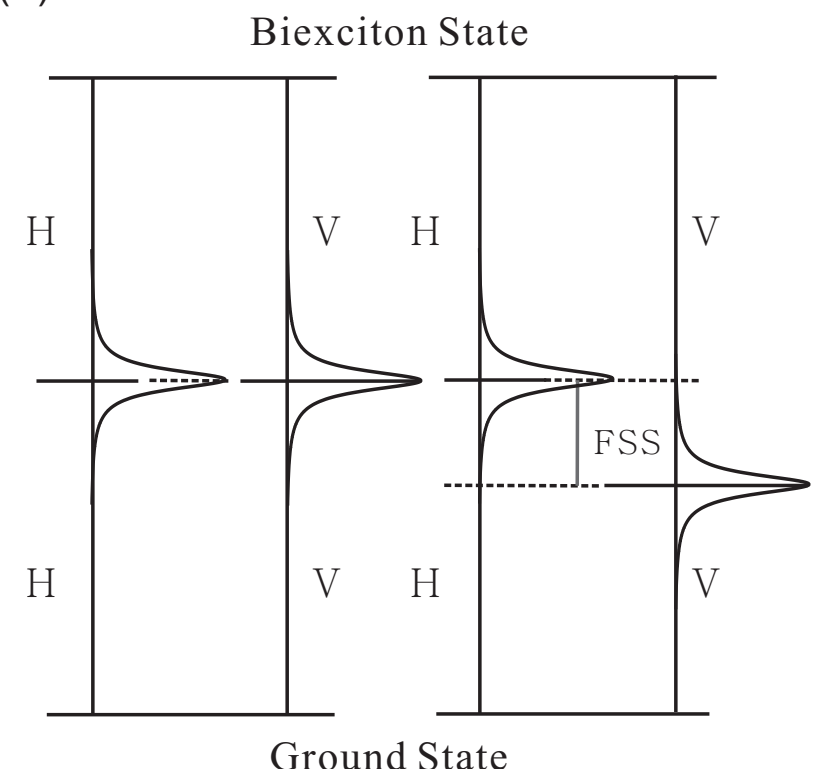

(b)

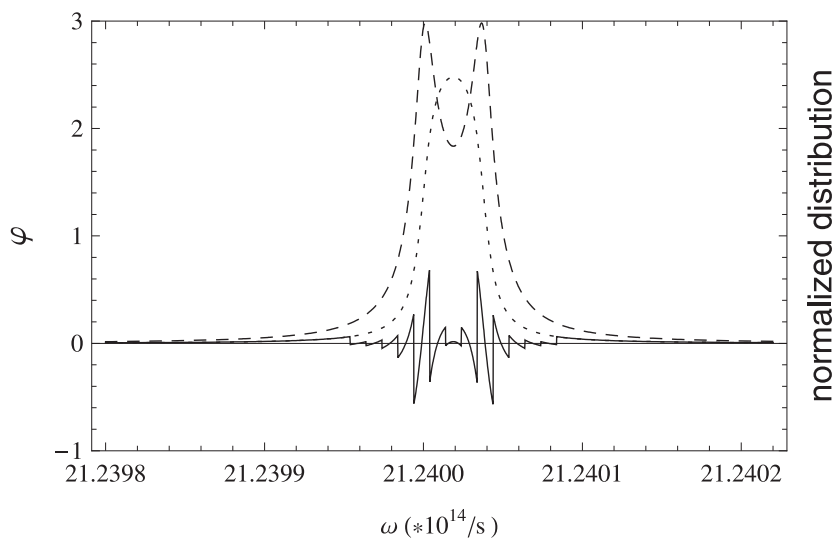

FIG. 1: (a) The level diagram of the radiative decay of the biexciton state. In the left panel, the FSS energy $S$ of the QD is zero, while in the right $S$ is nonzero. (b) A composite graph of the normalized $f_{H}(\omega) f_{V}(\omega)$ distribution (dashed line) and the phase difference between $\mathrm{H}$ and $\mathrm{V}$ polarization (dotted line) as a function of angular frequency $\omega$ with $S$ of $2.5 \mathrm{meV}$. The solid line shows the phase after compensation as discussed in the text.

the use of a dichroic mirror (DM) to separate them. The emitted XX photon goes directly to a single photon detector (SPD). The $X$ photon enters a polarization beam splitter (PBS), which reflects the $\mathrm{V}$ polarization and transmits the $\mathrm{H}$ polarization. The two parallel gratings distribute the photons in a spatial mode depending on their wavelengths. The diffraction angle $\theta$ is determined by $d \sin \theta-d \sin i=\lambda$, where $i$ is the incident angle and $\lambda$ is the photon's wavelength. The last mirror reflects the photon back. The quarter-wave plate (QWP) is $22.5^{\circ}$ placed. Passing through the QWP twice changes H (V) polar- ization to $\mathrm{V}(\mathrm{H})$ polarization. The half-wave plate (HWP) and the polarizer placed before the detectors are used to choose the polarization state for coincidence detection. All gratings, incident angles, and optical paths are identical in both arms of $\mathrm{H}$ and $\mathrm{V}$ polarization. As shown in Fig. 1(b), the phase difference is driven close to zero after which is divided in many small steps. Here each step corresponds to an angular frequency bandwidth of $\Delta \omega \doteq 1 \times 10^{10} / \mathrm{s}$. Some parameters have been chosen, the vertical distance between the two parallel gratings is 0.29 meters, the gratings' constant $d=1.1 \mu \mathrm{m}$, and the incident angles satisfy $\sin i=0.18$. The angular frequency $\omega=2.124 \times 10^{15} / \mathrm{s}$, corresponds to a wavelength of $0.887 \mu \mathrm{m}$. We choose the length of each step in front of the mirror as $\Delta=20 \mu \mathrm{m}$, which is much larger than the photon wavelengths.

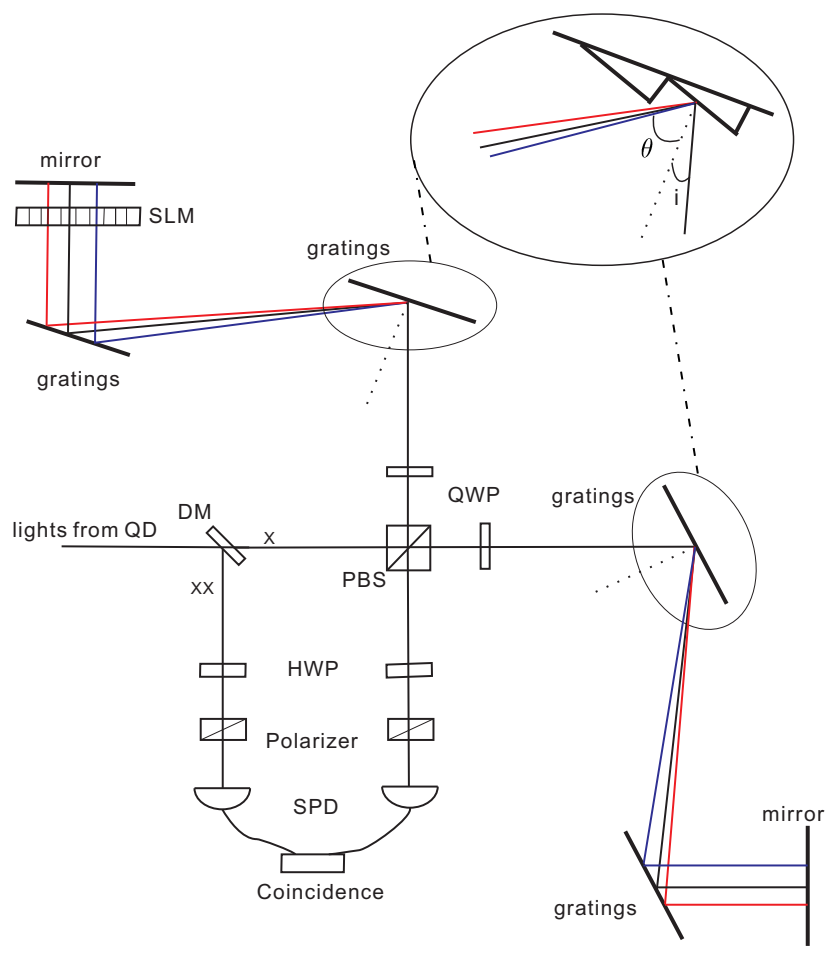

FIG. 2: Experimental setup for entanglement phase compensation. $\mathrm{X}$ and $\mathrm{XX}$ photons are separated by the DM. The XX photons go directly to SPD, while the $X$ photons are separated into two arms by PBS. To realize the phase compensation, a SLM is inserted in one of the paths.

Based on equation (4), the variation fidelity with FSS energy is shown in Fig. 3, where the dotted line corresponds to the phase difference $\varphi$ as mentioned above, and produces exactly the same results as obtained in the time domain [18]. The solid line corresponds to the ideal case without phase difference (i.e, $\varphi=0$ ). Here the QD exciton lifetime $\tau$ is set to $0.77 \mathrm{~ns}$ which is consistent with experimental observations [18].

Several methods can be used to realize this sectionalized compensation spatially, such as an optical coating with varying thickness, a medium of varying refractive index, or Fiber 
Bragg Grating (FBG) which is the standard dispersion compensation techniques used in optical fiber communications [21]. However the more appropriate method would be to use a phase-only spatial light modulator (SLM), which can change the phase delay distribution spatially pixel by pixel with an electric signal. This is important in this scheme as different QDs have different FSS energies, and hence their phase distributions differ. The advantages is that while other methods may require completely new fabrication to adapt to certain QDs, here with a SLM, different QDs just require changes in the electric signal for each pixel of the SLM. The phase range shown in Fig. 1(b) can never be larger than $\pi$, therefore the phase compensation can be easily realized with a SLM. Moreover, SLMs with pixel resolution of $20 \mu \mathrm{m}$ are commercially available, and even $5 \mu \mathrm{m}$ resolution can be achieved. A higher resolution will give finer compensations, and thus obtain the fidelity closer to the ideal altough at the expense of photon loss due to diffraction. With $20 \mu \mathrm{m}$ length steps, corresponding to the bandwidth $\Delta \omega \doteq 1 \times 10^{10} / \mathrm{s}$, the result is already very close to the ideal case as shown in Fig. 3. The dashed line gives the result after phase compensation and the solid line shows the ideal case when $\varphi=0$. Furthermore, for $\Delta=20 \mu \mathrm{m}$, diffraction effects are negligible. The photon loss caused by diffraction can be estimated as $\lambda / \Delta$, which is vanishingly small in this case.

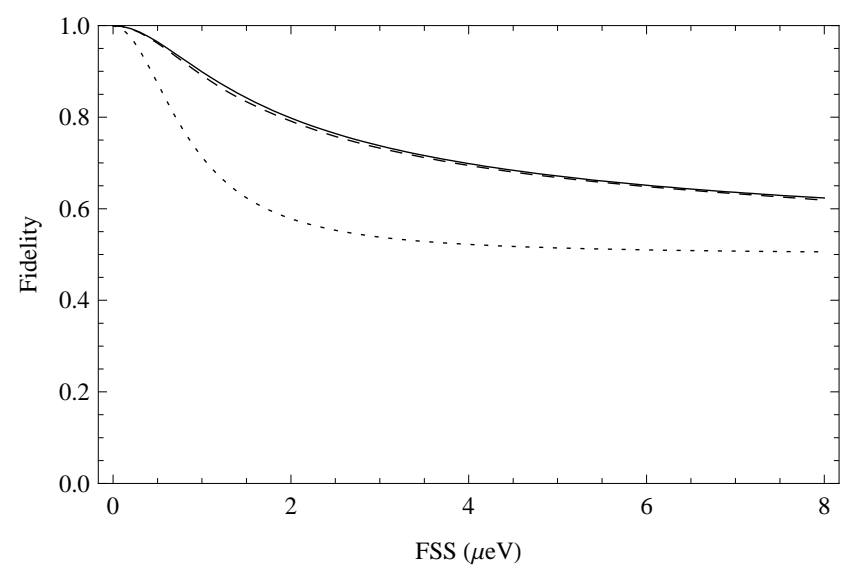

FIG. 3: The variation of fidelity with FSS energy. The dotted line is without phase compensation. The solid line shows the ideal case without any phase difference while the dashed line gives the results with phase compensation as mentioned in the text.

As shown in Fig. 3, with a FSS energy $S$ of $2.5 \mu \mathrm{eV}$, the fidelity increases from 0.553 to 0.764 after phase compensation. Even with $S$ of $3.8 \mu \mathrm{ev}$, the fidelity is still over 0.7 after phase compensation. We have noted that in a published report that with $S$ of $2.5 \mu \mathrm{eV}$ obtained by applying a timing gate, the fidelity increases from 0.46 with a gate width of $2 \mathrm{~ns}$ to 0.73 with a gate width of $49 \mathrm{ps}$ [19]. A simple calculation gives a theoretical collective efficiency of 0.925 with the $2 \mathrm{~ns}$ gate, but rapidly declines down to 0.061 with the 49 ps gate.
It is obvious that to get higher fidelity more photons have to be rejected by the timing gate. In contrast, we improve the fidelity by a factor of 0.21 without any photon loss theoretically. Considering the practical performance of the gratings (efficiency 90\%) and the SLM (efficiency 95\% ), we estimate the experimental efficiency of $62 \%$ in performing phase compensation. The bare postselection in energy [16] is even more wasteful than applying a timing gate [19], since one has there to select a small fraction of photons with overlapping frequencies, and further ensure that the phase difference does not change much in the selected frequency band.

Even after phase compensation, the fidelity unfortunately cannot attain unity, because it is limited by the photons outside the overlapping part of the frequency. Further improvements in fidelity can be achieved by rejecting these photons. For example, an even better performance than that of the ideal case shown with solid line in Fig. 3, can be achieved in cooperation energy-resolved postselection as reported in Ref. [16]. With phase compensation, this postselection can be more efficient and result in a much enhanced performance in fidelity. To illustrate the point, let $\mathrm{S}=2 \mu \mathrm{eV}$, if the angular frequency bandwidth is set at $\left\{2.1240006 \times 10^{15} / \mathrm{s}, 2.1240024 \times 10^{15} / \mathrm{s}\right\}$, then after phase compensation, the fidelity increases from 0.578 to 0.9 with a theoretical efficiency of 0.2 (still much higher than that obtained by applying a timing gate). Our result means that if a relative low efficiency can be tolerated, even a non-zero FSS is acceptable and required no magnetic or other fields to tune the FSS to zero, thus greatly simplifying the experimental setup. As reported previously [22], calculations reveal that InAs/InP QDs offer smaller FSSs with only a little flux around zero for individual QDs. Very recently, Mohan et al. [17] reported that highly symmetric, site-controlled quantum dots show FSS energies of several $\mu \mathrm{eV}$. Utilizing these types of QDs, our scheme may lead to a practical entangled photon pairs source that is efficient and easy to control.

Another advantage of this setup is that it is easy to control the output phase, an aspect that is highly desirable in a multitude of contexts in quantum information processings. This is achieved simply by either allowing the SLM to introduce a constant delay, or changing the optical path in one of the arms.

It should be noticed that we have not considered the effect of spin flipping and background light here, which in practice may slightly degrade the experimental results. Further studies would need to include the evolution of these effects and others, and try to solve them in the frequency domain.

To summarize, we have analyzed the degradation in entanglement of photon pairs emitted from QDs with non-zero FSS, and proposed a phase compensation scheme with the insertion of a SLM to greatly enhance entanglement gaining theoretical efficiencies approaching $100 \%$. An even better performance in fidelity can be achieved in cooperation with frequency postselection.

This work was supported by National Fundamental Research Program, National Natural Science Foundation of China (Grant No. 60621064, 10874162, and 10734060). 
[1] Special issue of Proc. R. Soc. London A 454, No.2921 (1998).

[2] A. K. Ekert, Phys. Rev. Lett. 67, 661 (1991).

[3] C. H. Bennett, and S. J. Wiesner, Phys. Rev. Lett. 69, 2881 (1992).

[4] C. H. Bennett, G. Brassard, C. Crépeau, R. Jozsa, A. Peres, and William K. Wootters, Phys. Rev. Lett. 70, 1895 (1993). D. Boschi, S. Branca, F. De Martini, L. Hardy, and S. Popescu, Phys. Rev. Lett. 80, 1121 (1998).

[5] Andrew G. White, Daniel F. V. James, Philippe H. Eberhard, and Paul G. Kwiat, Phys. Rev. Lett. 83, 3103 (1999).

[6] Jin-Shi Xu, Chuan-Feng Li, and Guang-Can Guo, Phys. Rev. A 74, 052311 (2006).

[7] Oliver Benson, Charles Santori, Matthew Pelton, and Yoshihisa Yamamoto, Phys. Rev. Lett. 84, 2513 (2000).

[8] Charles Santori, David Fattal, Matthew Pelton, Glenn S. Solomon, and Yoshihisa Yamamoto, Phys. Rev. B. 66, 045308 (2002).

[9] R. J. Young, R. M. Stevenson, A. J. Shields, P. Atkinson, K. Cooper, D. A. Ritchie, K. M. Groom, A. I. Tartakovskii, and M. S. Skolnick, Phys. Rev. B. 72, 113305 (2005).

[10] B.D. Gerardot, S. Seidl, P.A. Daigarno, R. J. Warburton, D. Granados, J.M. Garcia, K. Kowalik, and O. Krebs, Appl. Phys. Lett, 90, 041101 (2007).

[11] R. M. Stevenson, R. J. Young, P. See, D. G. Gevaux, K. Cooper, P. Atkinson, I. Farrer, D. A. Ritchie, and A. J. Shiedlds, Phys. Rev. B. 73, 033306 (2006).

[12] R. M. Stevenson, R. J. Young, P. Atkinson, K. Cooper, D. A.
Ritchie, and A. J. Shiedlds, Nature 439, 12 (2006).

[13] S. Seidl, M. kroner, A. Högele, K. Karrai, R. J. Warbuton, A. Badolato, and P. M. Petroff, Appl. Rhys. Lett. 88, 203113 (2006).

[14] Andreas Muller, Wei Fang, John Lawall, and G. S. Solomon, Phys. Rev. Lett. 103, 217402 (2009).

[15] R. Hafenbrak, S. M. Ulrich, P Michler, L Wang, A Rastelli and O. G. Schmidt, New J. Physics. 9,315 (2007).

[16] N. Akopian, N. H. Lindner, E Poem, Y. Berlatzky, J. Avron, D. Gershoni, B. D. Gerardot and P. M. Petroff, Phys. Rev. Lett. 96, 130501 (2006).

[17] A. Mohan, M. Felici, P. Gallo, B. Dwir, A. Rudra, J. Faist, and E. Kapon, Nature Photonics (7 March 2010) doi:10.1038/nphoton.2010.2 Letter

[18] A. J. Hudson, R. M. Stevenson, A. J. Bennett, R. J. Young, C. A. Nicoll, P. Atkinson, K. Cooper, D. A. Ritchie, and A. J. Shields, Phys. Rev. Lett. 99, 266802 (2007).

[19] R. M. Stevenson, A. J. Hudson, A. J. Bennett, R. J. Young, C. A. Nicoll, D. A. Ritchie, and A. J. Shields, Phys. Rev. Lett. 101, 170501 (2008).

[20] Michio Ikezawa, Selvakumar V. Nair, Hong-Wen Ren, Yasuaki Masumoto, and Harry Ruda, Phys. Rev. B 73, 125321 (2006).

[21] K. O. Hill, F. Bilodeau, B. Malo, T. Kitagawa, S. Thériault, D. C. Johnson, and J. Albert, Opt. Lett. 19, 1314 (1994).

[22] Lixin He, Ming Gong, Chuan-Feng Li, Guang-Can Guo, and Alex Zunger, Phys. Rev. Lett. 101, 157405 (2008). 